Technical note

\title{
Integrated structure and control design for mechatronic systems with configuration-dependent dynamics
}

\author{
Maíra Martins da Silva ${ }^{\mathrm{a}, *}$, Olivier Brüls ${ }^{\mathrm{b}}$, Wim Desmet ${ }^{\mathrm{a}}$, Hendrik Van Brussel ${ }^{\mathrm{a}}$ \\ ${ }^{a}$ Katholieke Universiteit Leuven, Department of Mechanical Engineering, Celestijnenlaan 300 B, B-3001 Leuven, Belgium \\ ${ }^{\mathrm{b}}$ University of Liège, Department of Aerospace and Mechanical Engineering (LTAS), Chemin des Chevreuils 1, 4000 Liège, Belgium
}

\section{A R T I C L E I N F O}

\section{Article history:}

Received 28 November 2007

Accepted 9 June 2009

\section{Keywords:}

Virtual prototyping

Motion control

Flexible multibody systems

Multi-objective optimization

\begin{abstract}
A B S T R A C T
This paper considers the optimal design of mechatronic systems with configuration-dependent dynamics. An optimal mechatronic design requires that, among the structural and control parameters, an optimal choice has to be made with respect to design specifications in the different domains. Two main challenges are treated in this paper: the non-convex nature of the optimization problem and the difficulty in modeling serial machines with flexible components and their embedded controllers. The optimization problem is treated using the direct design strategy which considers simultaneously structural and control parameters as variables and adopts non-convex optimization algorithms. Linear time-invariant and gain-scheduling PID controllers are addressed. This methodology is exploited for the multi-objective optimization of a pick-and-place assembly robot with a gripper carried by a variable-length flexible beam. The resulting design tradeoffs between system accuracy and control efforts demonstrate the advantage of an integrated design approach for mechatronic systems with configuration-dependent dynamics.
\end{abstract}

(c) 2009 Elsevier Ltd. All rights reserved.

\section{Introduction}

An optimal mechatronic design requires that, among the structural and control parameters, an optimal choice has to be made with respect to design specifications in the different domains [1]. In spite of the advances in optimal control design, optimal mechatronic design is still an open research area. There are, mainly, two reasons for that: (i) the difficulty in solving optimization problems involving structural and control parameters due to their non-convex nature and (ii) the difficulty in modeling mechatronic systems due to their multidisciplinary nature.

Considering that $\mathbf{P}$ represents the structural system plant and $\mathbf{K}$ the control system, the integrated structure and control optimization problem can be described by the following optimization problem:

$\min _{\mathbf{s} \in \Omega} f\left(\mathbf{L}\left(\mathbf{s}_{p}, \mathbf{s}_{k}\right)\right)$

where $\mathbf{s}$ is the vector of structural, $\mathbf{s}_{p}$, and control, $\mathbf{s}_{k}$, variables; $\Omega$ is the feasible solution set and $f$ is a measure of the system dynamic response which depends on the open-loop transfer function $\mathbf{L}(\mathbf{s})=\mathbf{P}\left(\mathbf{s}_{p}\right) \mathbf{K}\left(\mathbf{s}_{k}\right)$. Among other issues, structural and control variables are multiplied in order to evaluate the open-loop transfer

\footnotetext{
* Corresponding author. Tel.: +32 163224 80; fax: +32 16322987

E-mail address: mairams@gmail.com (M.M. da Silva).
}

function, resulting into a non-convex optimization problem. There is no computationally tractable approach to solve Eq. (1) due to the complex and non-convex nature of the optimization [2]. According to [3], there are mainly two numerical strategies to perform the integrated structure/controller design: the nested and the direct strategies.

The nested design strategy combines nonlinear optimization methods and model-based control design techniques, such as the ones based on linear matrices inequalities (LMI) and Ricatti equations. In other words, for each set of structure parameters, a control, with a general structure, is designed using model-based control design techniques. The nested design strategy has been employed for designing a gain-scheduling control to achieve compensation for the varying mass distribution, to suppress structural bending, vibrations and friction disturbances, and to achieve shorter motion settling time [4]. Recently, [5] have proposed a general platform for designing serial and parallel machines based on the nested design strategy. The main drawback of this strategy is that the variables are not optimized simultaneously.

The direct design strategy considers simultaneously the control and structural parameters, using numerical methods, such as nonconvex optimization algorithms or genetic algorithms. It can be employed when the control structure is known beforehand. An important drawback of this approach is the excessive computation time, which grows exponentially with the number of structural design variables. The direct design strategy has been employed, 
among others, for optimizing a two-link planar manipulator and a PD controller [6], for optimizing the geometry and the control parameters of a motor-driven four-bar system [7], for optimizing structural and control parameters aiming cavity noise reduction [8] and for designing structural and control parameters of a flexible linkage mechanism for noise attenuation [9]. In contrast with the control strategies exploited in this paper, most references regarding direct design strategies do not address the design of linear parameter-varying (LPV) controllers. Because of the non-convex nature of the direct design strategy, a LPV system can not be described as a polytopic system [10]. This is an important drawback when designing LPV controllers using frequency-domain metrics, since the infinite set of inequalities imposed by the parameter variation can not be reduced to a finite one. For this reason, time-domain metrics are considered in this paper, which demands timedomain simulation and evaluation of the system under study.

Besides the complex and non-convex nature of the optimization problem, the integrate structure and control design is a challenge task due to the difficulty in modeling some mechatronic systems. This is the case of serial machines with flexible components, such as Cartesian mechanisms, milling machines and pick-and-place machines. In these machines, the relative motion between flexible components leads to time-varying boundary conditions, so that the eigenfrequencies and mode shapes are not constant but depend on the spatial configuration. The dynamic modeling of serial machines has been treated in recent references [11-14]. A substructuring dynamic modeling procedure has been applied for the modeling of a flexible-link planar parallel platform by [12] using Component Mode Synthesis (CMS) [15]. A similar procedure was employed in the modeling of a 3-axis milling machine by [11] for several discrete spatial configurations. However, CMS can not be directly employed to evaluate a serial machine in time-domain, since it is not possible to represent the flexibilities using a single mode set for each component due to their time-varying boundary conditions. Since the most available commercial multibody packages also use CMS to include flexibility, the modeling of serial machines is not a straightforward task. Alternatives have been proposed by $[13,14]$, integrating dedicated software such as commercial finite element and multibody packages. However, these techniques are relatively time-consuming.

This paper concerns the integrated design of serial machines with configuration-dependent dynamics considering the direct design strategy. Two control strategies are compared: linear timeinvariant (LTI) PID and gain-scheduling PID. To proper simulate and evaluate serial machines in time-domain, an innovative feature have been implemented in Oofelie, an open source finite element software [16]. This feature, refereed to as sliding joint, is not available in most commercial multibody packages. It allows the relative translation motion between flexible bodies. To simulate and evaluate gain-scheduling controllers, Oofelie capabilities have been extended to include controllers described by LPV state-space equations. Based on this mechatronic design tool, able to simulate serial machines and different controllers in time-domain, a multiobjective optimization strategy is proposed for the integrated design of the mechanical structure and the controller. This approach is very useful to evaluate tradeoffs among conflicting objectives in mechatronic applications. The selected control design approach does not directly guarantee stability, which should be accessed after the control derivation. To cope with this requirement, stability analysis is included as a set of constraints in the optimization problem.

The paper is organized as follows: the general methodology for the modeling, stability analysis and optimization of mechatronic systems with configuration-dependent dynamics is described in Section 2. The integrated design methodology is applied to an industrial 3-axis pick-and-place assembly robot. Section 3 presents the case study description, its mechanical modeling, as well as various control algorithms and stability analysis. In Section 4 , the integrated structure and control optimization is developed. The results demonstrate the benefits of the mechatronic design approach. Finally, some conclusions are drawn in Section 5.

\section{Modeling and optimization of mechatronic systems}

\subsection{Modeling}

This paper presents a methodology to model and simulate a serial machine system with configuration-dependent dynamics in time-domain, using nonlinear flexible multibody dynamics. The approach described in [17], which is a general and systematic technique for the simulation of articulated systems with rigid and flexible components, is selected. For mechatronic systems, an extension of those modeling methods is required to deal with the controller dynamics. One option is to use a coupled modeling approach, so that a monolithic time integrator can be used, and no weak coupling assumption is required $[18,19]$. This strongly coupled formulation has been adopted for the present developments.

According to [17], a flexible multibody system can be described using absolute nodal coordinates. Hence, each body is represented by a set of nodes and each node has its own translation and rotation coordinates. The various bodies of the system are interconnected by kinematical joints, which impose restrictions on their relative motion. If the nodal coordinates are gathered in a vector q, the joints are thus represented by a set of $m$ nonlinear kinematic constraints:

$\mathbf{\Phi}(\mathbf{q}, t)=\mathbf{0}$

According to the Lagrange multiplier technique, the formulation of the constrained equations of motion requires the introduction a $m \times 1$ vector of Lagrange multipliers $\lambda$.

The dynamics of the controller can be represented by a nonlinear state-space model with state variables $\mathbf{x}_{k}$ and control signal output variables $\mathbf{y}_{k}$. In this way, the dynamic equations of a mechatronic system consisting of a multibody model and a control system (see Fig. 1a), have the general structure:

$$
\begin{aligned}
& \mathbf{M}(\mathbf{q}) \ddot{\mathbf{q}}=\mathbf{g}(\mathbf{q}, \dot{\mathbf{q}}, \mathbf{w}, t)-\mathbf{B}^{T} \lambda+\mathbf{y}_{k} \\
& \mathbf{0}=\mathbf{\Phi}(\mathbf{q}, t) \\
& \dot{\mathbf{x}}_{k}=\mathbf{f}\left(\mathbf{x}_{k}, \mathbf{u}_{k}, t\right) \\
& \mathbf{y}_{k}=\mathbf{h}\left(\mathbf{x}_{k}, \mathbf{u}_{k}, t\right)
\end{aligned}
$$

Eq. (3) represents the dynamic equations of the mechanical system, Eq. (4) the kinematic constraints, Eq. (5) the state equation and Eq. (6) the output equation. $\mathbf{M}$ is the mass matrix, which is not constant in general, $\mathbf{g}$ represents the internal, external and complementary inertia forces, $\mathbf{B}=\partial \mathbf{\Phi} / \partial \mathbf{q}$ is the matrix of constraint gradients, $\mathbf{y}_{k}$ denotes the actuator forces or torques generated by the control action, $\mathbf{u}_{k}$ represents the input signals to the controller and $\mathbf{w}$ represents the disturbance, noise and reference signals vector. Eqs. (3)(6) are coupled equations of motion and can be solved numerically using an implicit time integration scheme. Typical applications are described in [18].

Fig. 1a shows a scheme of the augmented plant $\mathbf{P}_{a}$, which includes the mechanical system $\mathbf{P}$ described by Eqs. (3) and (4), and the control system $\mathbf{K}$ described by Eqs. (5) and (6). The notation is the same one adopted in Eqs. (3)-(6). The output system signal $\mathbf{z}$ and control signal inputs $\mathbf{u}_{k}$ can be described by combinations of the disturbance, noise and reference signals, $\mathbf{w}$, the control signal outputs, $\mathbf{y}_{k}$, and the measurements from the mechanical system, which can be positions $\mathbf{q}$, velocities $\dot{\mathbf{q}}$ or accelerations $\ddot{\mathbf{q}}$. The objective is to design a controller $\mathbf{K}$ that minimizes the signal z. Fig. 1b shows a scheme of the selected control strategy which is 

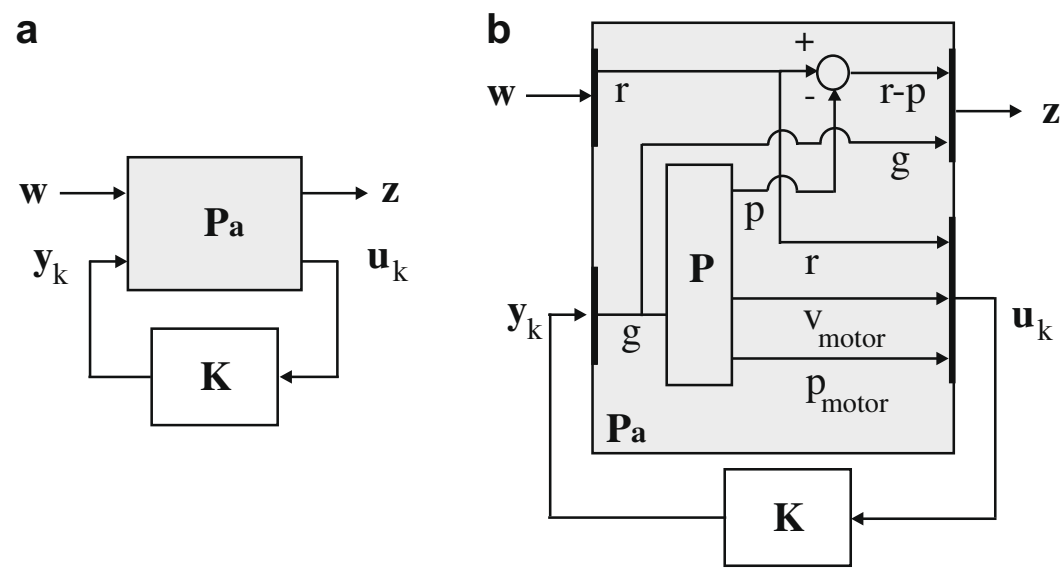

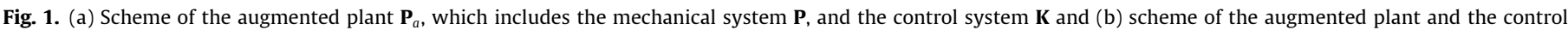
system of the case study described in Section 3.3.

described in Section 3.3. In this case, $\mathbf{w}$ regards only the reference signal, $r$, and $\mathbf{z}$ regards the tracking error, $r-p$, and the force generated by the controller, $g$. The gripper position is refereed as to $p$.

In the case of mechatronic systems with configuration-dependent dynamics, LPV controllers are widely employed $[4,5,20]$. Using the simulation framework described by Eqs. (3)-(6), LPV controllers can be included adapting the ABCD matrices according to time-varying parameters $\mathbf{l}(t)$ :

$$
\begin{aligned}
& \dot{\mathbf{x}}_{k}(t)=\mathbf{A}_{k}(\mathbf{l}(t)) \mathbf{x}_{k}(t)+\mathbf{B}_{k}(\mathbf{l}(t)) \mathbf{u}_{k}(t) \\
& \mathbf{y}_{k}(t)=\mathbf{C}_{k}(\mathbf{l}(t)) \mathbf{x}_{k}(t)+\mathbf{D}_{k}(\mathbf{l}(t)) \mathbf{u}_{k}(t)
\end{aligned}
$$

\subsection{Stability analysis}

When stability is not guaranteed directly by the control design approach, it needs to be, eventually, accessed. The stability analysis of LPV systems can be performed with Lyapunov-based theory. Recently, a sufficient condition for the stability of LPV systems has been provided by [21] taking into account a bound $\Delta$ on the rate of parameter variation. The system under verification should be described as a discrete-time LPV system $\mathbf{x}(i+1)=\mathbf{A}(\mathbf{l}(i)) \mathbf{x}(i)$, where the varying parameter described in continuous-time, $\mathbf{l}(t)$, is represented in different time steps $i$ by $\mathbf{l}(i)$.

For a given maximal rate of variation $\Delta$, the parameter space is divided into $v$ intervals. The size of the intervals is such that in one discrete-time step, the parameter $\mathbf{l}(i)$ can only jump into the next interval:

$\frac{|\mathbf{l}(i+1)-\mathbf{l}(i)|}{T_{s}} \leqslant \Delta$

where $T_{s}$ is the sample period and $i=0 \ldots v$. A simplified notation for the theorem presented in [21], which states a sufficient condition for stability of an LPV system, is proposed in [22] and described hereafter. Considering a discrete-time LPV system described by $\mathbf{x}(i+1)=\mathbf{A}(\mathbf{l}(i)) \mathbf{x}(i)$, if there exist $i=1 \ldots v$ positive definite constant matrices $\mathbf{P}(i)$, such that the following LMIs are satisfied for all $i=1 \ldots v$ and $j=-1,0,1$ :

$$
\begin{aligned}
& \mathbf{A}(\mathbf{l}(i))^{T} \mathbf{P}(i+j) \mathbf{A}(\mathbf{l}(i))-\mathbf{P}(i)<0 \\
& \mathbf{A}(\mathbf{l}(i+1))^{T} \mathbf{P}(i+j) \mathbf{A}(\mathbf{l}(i+1))-\mathbf{P}(i)<0
\end{aligned}
$$

then the system is uniformly asymptotically stable for all timevarying realization of the parameter $\mathbf{1}$, satisfying constraints on the range and rate of the parameter variation. Due to the notation simplification, the first LMI of the first interval and the last LMI of the last interval are not valid and should be removed.

\subsection{Multi-objective optimization}

The objective of this work is to perform a direct optimization considering structural and control parameter. As stated in the Introduction, this is a non-convex and non-linear optimization (Eq. (1)). Moreover, this optimization problem is usually composed of distinct objectives, such as minimizing the tracking error and minimizing the motor effort. The latter characteristic suggests that multi-objective optimization strategies, which aim to find tradeoffs among several conflicting objectives, should be considered.

In the context of mechatronic systems, the vector $\mathbf{s}$ of design variables may include structural as well as control parameters. The optimization problem can be stated as

$\min _{\mathbf{s} \in \Omega} f_{i}(\mathbf{s}) \quad i=1, \ldots, n_{f}$

where $f_{i}\left(i=1, . ., n_{f}\right)$ denotes the set of objective functions, whereas $\Omega$ is the feasible solution set defined by the inequality constraints

$h_{i}(\mathbf{s}) \leqslant 0 \quad i=1, \ldots, n_{h}$

Let us note that the functions $f_{i}$ and $h_{i}$ are typically computed from simulation results. The objective functions evaluate the dynamic system performance addressed by the non-convex optimization problem (Eq. (1)).

An attempt to solve this non-convex, non-linear, multi-objective and computationally demanding optimization problem can be performed using stochastic methods, such as evolutionary algorithms. Among evolutionary algorithms, the Non-Dominated Sorting Genetic Algorithm proposed by [23] has been improved by [24] yielding an algorithm used for multi-objective problems: NSGA-II. In this refined algorithm, the population is ranked according to the individual's non-domination criterion before the selection. In other words, an individual is compared with every other individual in the present population and also with the non-dominated individuals from the previous population to find if it is dominated. Eventually, a large fitness value is assigned to the non-dominated solutions. This process is repeated to find the subsequent non-dominated solutions and it stops when all individuals in the present population are dominated by the non-dominated individuals from the previous population yielding the Pareto-optimal solutions. The diversity of the population is preserved by a crowded-comparison approach, which guarantees a good spread of solutions. 


\section{Pick-and-place robot: modeling details and control algorithms}

\subsection{Case study}

This integrated design methodology is applied to an industrial 3-axis pick-and-place assembly robot with a gripper carried by a flexible beam (Fig. 2). The fast movements of this machine may excite the vibrations of the variable-length flexible beam. The $Z$ and the $X$-motion are performed by a ACM H-drive system, which is cartesian robot based on three linear motor motion system, produced by Philips. The $Z$-motion is gantry driven by two linear motors and the $X$-motion, over the carriage, is also driven by a linear motor. The vertical $Y$-motion is actuated by a rotary brushless DCmotor which drives a vertical flexible beam by a ball screw/nut combination. The position of the linear motors and the beam length are measured by optical encoders, and the acceleration at the gripper in the $X$-direction is measured by an accelerometer. The objective is to move the gripper as accurately and fast as possible along a prescribed trajectory in the working area.

A model has been built to simulate the pick-and-place assembly robot motion in $X$ - and $Y$-directions. The $Z$-motion is not considered in this work.

\subsection{Mechanical model}

A flexible multibody model has been built to simulate the pickand-place robot motion in $X$ - and $Y$-directions (see Fig. 2) according to Eqs. (3) and (4). All components are modeled as rigid bodies, excepted the flexible beam. The inertia of the sliders of the linear motors, responsible for the $Z$-direction motion, is added to the bodies representing the frames; the gripper is modeled as a concentrated mass. Table 1 shows the inertia values for all rigid bodies.

A specific feature, a sliding joint, has been implemented to enable the translational relative motion between flexible bodies. This

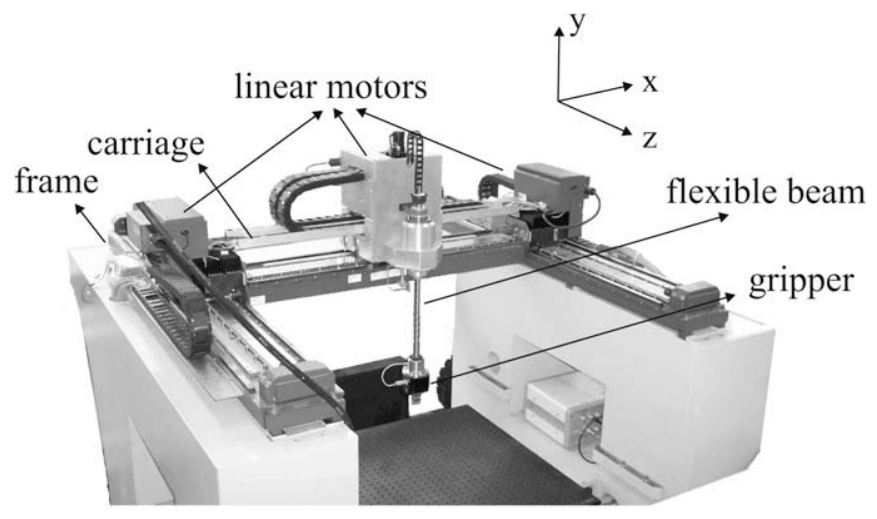

Fig. 2. Pick-and-place machine used as test-case

Table 1

Inertia values of the rigid bodies

\begin{tabular}{|c|c|c|c|c|c|c|c|}
\hline \multirow{2}{*}{$\begin{array}{l}\text { Rigid } \\
\text { bodies }\end{array}$} & \multirow{2}{*}{$\begin{array}{l}\text { Mass } \\
(\mathrm{kg})\end{array}$} & \multicolumn{3}{|c|}{ Moment of inertia $\left(\mathrm{kg} \mathrm{m}^{2}\right)$} & \multicolumn{3}{|c|}{ Center of gravity $(m)$} \\
\hline & & $I_{x x}$ & $I_{y y}$ & $I_{z z}$ & $x$ & $y$ & $z$ \\
\hline Frames & 169.0 & $1.0 \times 10^{3}$ & $2.0 \times 10^{3}$ & $1.0 \times 10^{3}$ & \pm 0.57 & 0.53 & 0.00 \\
\hline Carriage & 13.9 & $1.0 \times 10^{2}$ & $1.0 \times 10^{1}$ & $1.0 \times 10^{2}$ & 0.00 & 0.53 & 0.00 \\
\hline Linear motor & 31.0 & - & - & - & 0.00 & 0.53 & 0.00 \\
\hline Gripper & 1.25 & - & - & - & 0.00 & 0.00 & 0.00 \\
\hline
\end{tabular}

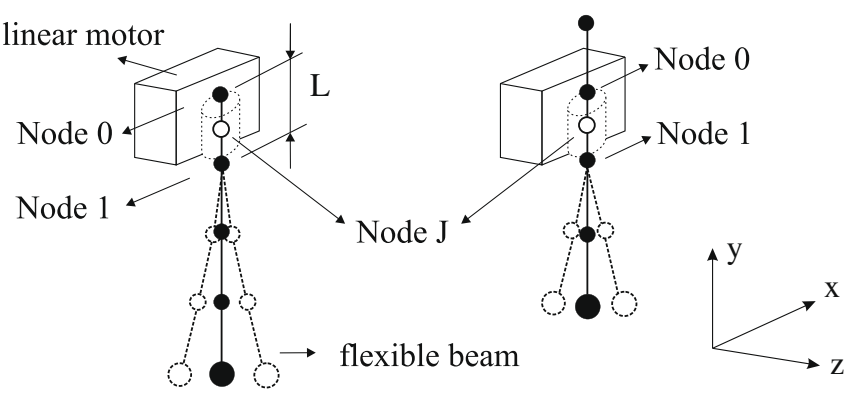

Fig. 3. Scheme of the translational movement between the linear motor and the flexible beam.

sliding joint is responsible for the translational motion in $Y$-direction between the flexible beam and the linear motor (see Fig. 3). According to the Timoshenko theory, displacements $\Gamma$ and rotations $\boldsymbol{\Psi}$ are treated as independent fields in the beam. In a single element with two nodes $n_{0}$ and $n_{1}$, an arbitrary point can be represented by the adimensional coordinate along the beam $\eta \in[0,1]$. For linear shape functions and under the assumption of small rotations, the positions and orientations of this point are expressed in terms of the nodal coordinates

$\boldsymbol{\Gamma}(\eta)=(1-\eta) \boldsymbol{\Gamma}_{0}+\eta \boldsymbol{\Gamma}_{1}$

$\boldsymbol{\Psi}(\eta)=(1-\eta) \boldsymbol{\Psi}_{0}+\eta \boldsymbol{\Psi}_{1}$

Using again the small rotations assumption, the axis of the beam and of the sliding joint are close to the $y$-axis. If the linear motor is represented by a node $J$, the sliding joint is thus modeled by five kinematic constraints between the nodal coordinates $\boldsymbol{\Gamma}_{0}=\left[\begin{array}{lll}x_{0} & y_{0} & z_{0}\end{array}\right]^{T}, \boldsymbol{\Gamma}_{1}=\left[\begin{array}{lll}x_{1} & y_{1} & z_{1}\end{array}\right]^{T}, \boldsymbol{\Gamma}_{J}=\left[\begin{array}{lll}x_{J} & y_{J} & z_{J}\end{array}\right]^{T}, \boldsymbol{\Psi}_{1}, \boldsymbol{\Psi}_{2}$ and $\boldsymbol{\Psi}_{J}$

$\boldsymbol{\Phi}_{1}=(1-\eta) x_{0}+\eta x_{1}-x_{J}=0$

$\boldsymbol{\Phi}_{2}=(1-\eta) z_{0}+\eta z_{1}-z_{J}=0$

$\boldsymbol{\Phi}_{3,4,5}=(1-\eta) \boldsymbol{\Psi}_{0}+\eta \boldsymbol{\Psi}_{1}-\boldsymbol{\Psi}_{J}=\mathbf{0}$

where $\eta$ is computed as $\eta=\left(y_{0}-y_{J}\right) / L$, with $L$, the total length of the beam element. Fig. 3 shows a scheme of the linear motor (rigid body) and the flexible beam (flexible body) in two configurations illustrating their behavior during the translational motion in $Y$ direction. The complete derivation of this feature can be found in [25].

A general scheme of the pick-and-place robot model is shown in Fig. 4. The actuator force generated by the linear motor, is applied to the linear motor mass (action) and to the carriage (reaction). The nominal machine specifications are described hereafter. The spring stiffness and the damping value between the carriage and the frame are, respectively, $K_{1}=9.15 \times 10^{6} \mathrm{~N} / \mathrm{m}$ and $D_{1}=1042 \mathrm{~N} \mathrm{~s} / \mathrm{m}$. The frame suspension is connected to the ground by four connecting points. The stiffness and the damping of these connections are, respectively, $K_{2}=5.3 \times 10^{7} \mathrm{~N} / \mathrm{m}$ and $D_{2}=5204 \mathrm{~N} \mathrm{~s} / \mathrm{m}$. The damping $D_{3}=100 \mathrm{~N} \mathrm{~s} / \mathrm{m}$ represents the connection between the linear motor and the carriage. The flexible beam has a nominal diameter of $24 \mathrm{~mm}$. The material properties are: density $\rho_{\mathrm{s}}=7800 \mathrm{~kg} / \mathrm{m}^{3}$, Poisson's Ratio $v=0.3$, damping ratio 0.01 and elasticity modulus $E=2.1 \times 10^{11} \mathrm{~N} / \mathrm{m}^{2}$. The mass and inertia values can be found in the machine manual. The stiffness values have been adjusted according to experimental data (Fig. 5)

This flexible multibody model has hundreds degrees-of-freedom and its dynamics varies according to the configuration. Thus, a full description of the dynamic equations can not be directly included in this section. For sake of completeness, a reduced model extracted from the full flexible multibody for a given configuration $(l=0.53 \mathrm{~m})$ is presented in Appendix A. Alternatively, a lumped model has been proposed by [26]. This lumped model can be 


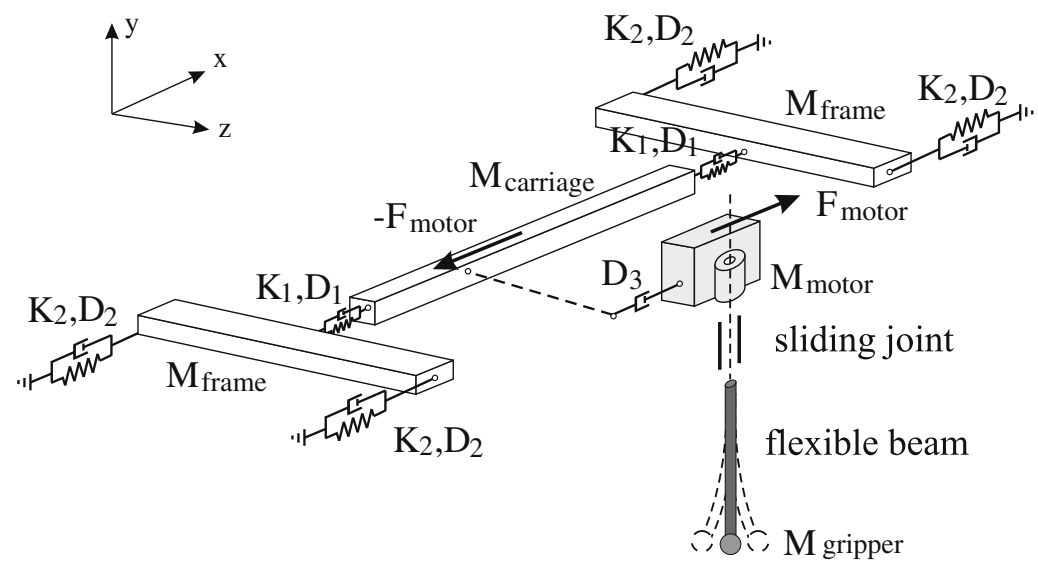

Fig. 4. Scheme of the flexible multibody model of the $X$-direction motion of a pick-and-place machine.
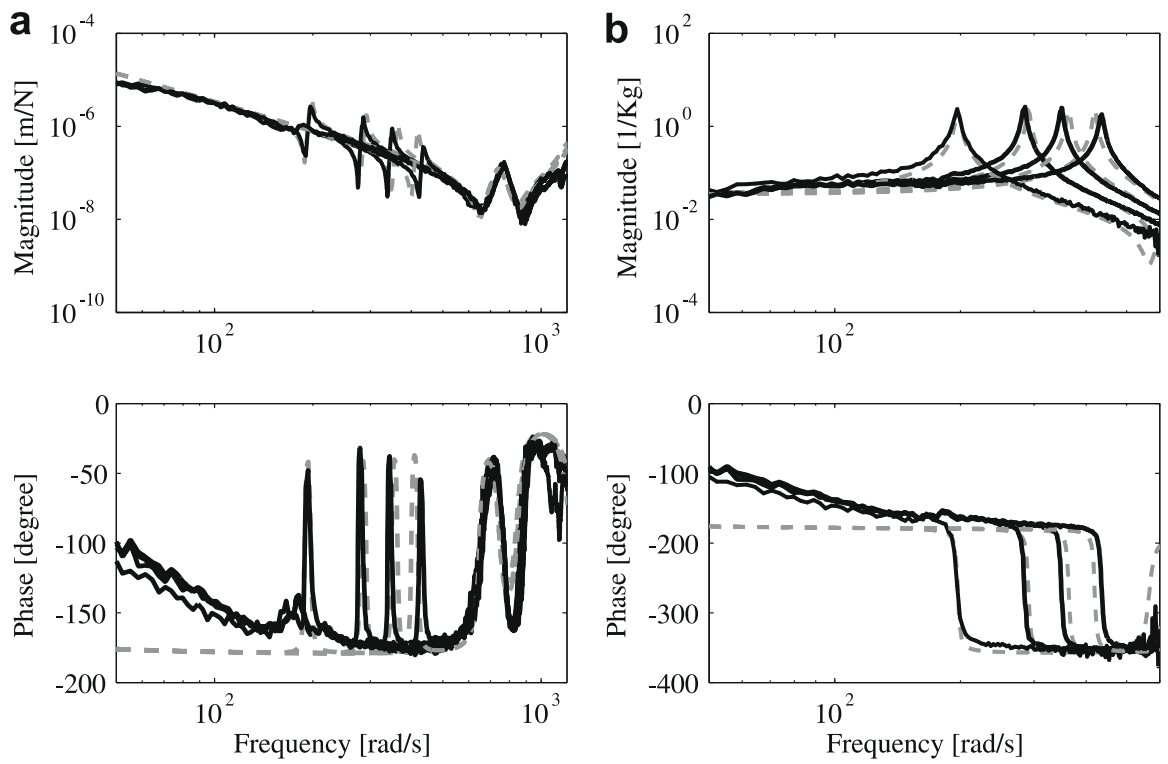

Fig. 5. Comparison between the simulated (- - dashed line) and the experimental FRFs (- full line). (a) Motor position/motor force (b) gripper acceleration/motor force.

employed for the evaluation of the nominal system over the configuration space.

Fig. 5 shows the comparison between simulated and measured FRFs for four beam lengths $(l=0.53,0.41,0.36$ and $0.33 \mathrm{~m}$ with the first resonance frequency at 32, 46, 57 and $63 \mathrm{~Hz}$ ). The curves are in a good agreement, which confirms the validity of the model.

\subsection{Controller}

For machines with configuration-dependent dynamics, two control strategies can be adopted: (1) LTI controllers, that can be explicitly designed to take into account the dynamic variations as uncertainties, like robust controllers designed using $\mu$-synthesis or (2) linear time-varying (LTV) controllers, that can adapt according to the parameter variations, such as LPV controllers [4] and LPV gain-scheduling controllers [20].

In this work, a PID control scheme is implemented for controlling the $X$-axis motion using measurements of the motor position. Both LTI and LPV gain-scheduling PID controllers are described by the following state-space representation

$\dot{\mathbf{x}}_{k}=[0] \mathbf{x}_{k}+[-110] \mathbf{u}_{k}$
$\mathbf{y}_{k}=\left[-K_{I}(\mathbf{l})\right] \mathbf{x}_{k}+\left[K_{P}(\mathbf{l})-K_{P}(\mathbf{l})-K_{D}(\mathbf{l})\right] \mathbf{u}_{k}$

where the input vector $\mathbf{u}_{k}=\left[r, p_{\text {motor }}, v_{\text {motor }}\right]$ collects the reference input, the motor position and the motor velocity; and the output vector $\mathbf{y}_{k}=[g]$ represents the motor force. The gripper position is refereed to as $p$. The performance of the system is measured by the gripper position accuracy.

As it can be observed in Fig. 5, the relation between the first resonance and the beam length is rather linear $(l=0.53,0.41,0.36$ and $0.33 \mathrm{~m}$ with the first resonance frequency at 32, 46, 57 and $63 \mathrm{~Hz}$ ). Therefore, linear dependence on $l$ is selected for the gain-scheduling PID. In this way, the vector of scheduling parameters $\mathbf{I}$ simply represents the beam length $\mathbf{l}=[l(t)]$.

Fig. $1 \mathrm{~b}$ shows a scheme of the augmented plant $\mathbf{P}_{a}$, which includes the mechanical system $\mathbf{P}$, and the control system $\mathbf{K}$. In this case, the signal $\mathbf{w}$ represents the reference signal, $r$; and the signal $\mathbf{z}$ represents the tracking error, $r-p$, and the actuation force generated by the controller, $g$. The control signal inputs $\mathbf{u}_{k}$ are $\left[r, p_{\text {motor }}, v_{\text {motor }}\right]$.

The same strategy can be applied for modeling and controlling the Z-direction motions, but this design is not considered in this work. An imposed motion assures that the $Y$-direction motion follows the prescribed trajectory. 


\subsection{Stability analysis}

A state-space description of the machine model, for a given configuration, is obtained by using a model-order reduction technique [27]. For $l=0.53 \mathrm{~m}$, the state-space model is described in the Appendix A. This state-space model is then combined, in a feedback fashion, with the state-space description of the PID controller (Eqs. (22) and (18)), yielding the closed-loop state-space model of the system with its embedded controllers for a given configuration. This procedure should be repeated for each configuration considered during the stability analysis.

These closed-loop state-space models are then discretized considering a sampling time of $1 / 2000 \mathrm{~s}$. The frequency range of interest is from 0 to $400 \mathrm{~Hz}$, where the first three system resonances are located. In this way, the sampling rate can guarantee good measurements, if they are eventually performed, in the frequency range of interest.

In order to guarantee that the system is uniformly asymptotically stable for a parameter variation between 0.33 and $0.53 \mathrm{~m}$ (the beam length) and bounded rate by $10.0 \mathrm{~m} / \mathrm{s}$, the parameter space should be discretized in $v=40$ intervals $(l(i+1)-l(i)<$ 0.005). The feasibility problem described by the LMIs (in Eq. (9)) can be solved using the LMI toolbox available in Matlab [28]. Since 40 intervals should be evaluated, 41 closed-loop state-space models should be extracted for each configuration considered (between 0.33 and $0.53 \mathrm{~m}$ ).

\section{Integrated structure and control design}

In pick-and-place applications, the position error should be kept below a specified threshold. The diameter of the beam has a direct influence on the vibration of the effector and is thus considered as a design variable. The other parameters are associated with the particular PID control strategy, as described below. The set of variables is collected in the vector of design variables noted $\mathbf{s}$.

In order to mimic point-to-point movements, a pulse train is chosen as a reference signal for the optimization problem. As illustrated in Fig. 6 , the beam length, $l(t)$, evolves during the simulation, so that the different eigenfrequencies are excited in various configurations. For this reference input, the simulation takes about $110 \mathrm{~s}$ CPU time using a Pentium IV, with a processor of $1.4 \mathrm{GHz}$.

Taking these aspects into account, the minimization of two objective functions is considered.
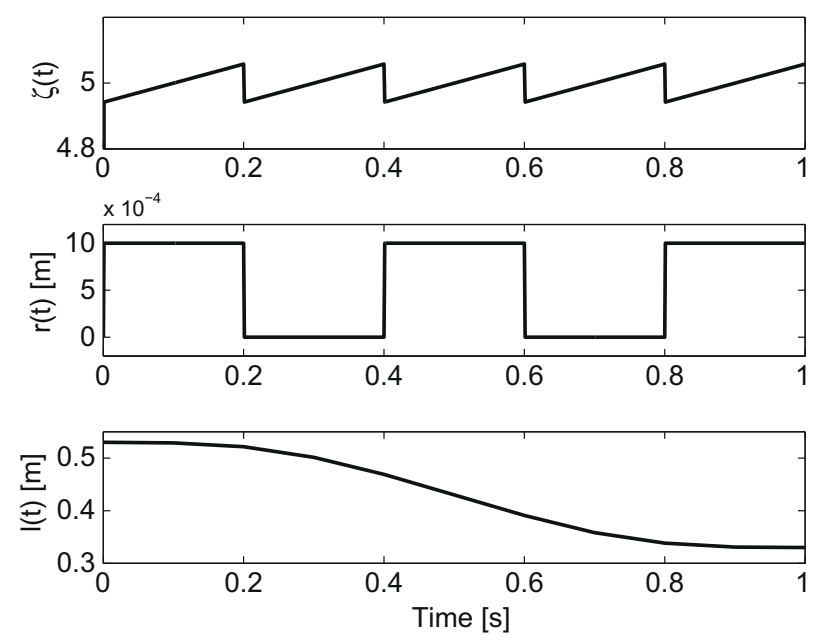

Fig. 6. The weighting function $\zeta(t)$, the reference input $r(t)$ and beam length variation during the simulation $\mathrm{l}(\mathrm{t})$.
The first objective function $f_{1}$ represents the weighted squared error between the gripper position, $p(t, \mathbf{s})$, and the reference signal, $r(t)$, and is computed according to

$f_{1}(\mathbf{s})=\frac{1}{c_{e}} \int_{0}^{1} \zeta(t)(r(t)-p(t, \mathbf{s}))^{2} d t$

where $t$ refers to time, $c_{e}=4 \times 10^{-4}$ is a constant and $\zeta(t)$ is a weighting function. The value of constant $c_{e}$ is chosen in such a way that the values of $f_{1}$ are normalized between 0 and 1 . Too large or too small objective or constraint values can imply in numerical errors during the optimization procedure. Therefore, it is a common practice to normalize these values.

The weighting function $\zeta(t)$, shown in Fig. 6 , is adopted to penalize longer settling times. A basic weighting function, $\zeta^{*}(t)=$ $\tan (\alpha) * t+1 / T-\tan (\alpha) * T / 2$, where $t$ is the time within each step interval and $T$ represents the total simulation time associated with the step input (in the present case $0.2 \mathrm{~s}$ ) and $\alpha$ is the curve steepness. An illustration of this basic weighting function is depicted in Fig. 7a. An attempt to define such weighting functions is addressed in [6], but no clear guidelines are proposed. In the way it is presented here, the angle $\alpha$ can be adjust such that the weighting function penalizes more large overshoot or longer settling time. An example illustrates the behavior of this basic weighting function for a second-order system with bandwidth of $10 \mathrm{~Hz}$ and a damping factor of 0.01 . The reference input (a step) and the system response are shown in Fig. 7b and c, respectively. Fig. 7d and e show two different weighting functions and the respective weighted squared errors. The higher $\alpha$, the lower is the penalty on the overshoot and the higher is the penalty on the settling time. For the case study presented in this work, the angle $\alpha$ is chosen to be $60^{\circ}$, illustrated in Fig. 6 , which penalizes longer settling times.

The second objective function $f_{2}$ represents the maximum force $(g)$ required by the controller during motion, i.e.

$f_{2}(\mathbf{s})=\frac{\max |g(t, \mathbf{s})|}{c_{f}}$

where $c_{f}=500 \mathrm{~N}$ is a constant for normalizing $f_{2}$. The maximum force delivered by the present motor is $500 \mathrm{~N}$, which motivates the $c_{f}$ value choice. Normally, a linear motor is selected based on the maximum force required by the controller to perform a desired motion. Since this is an expensive item, the present motor is kept, enforcing that the maximum required force is below $500 \mathrm{~N}$. In this way, the values of $f_{2}$ can only vary between 0 and 1 (normalized objective), which will be enforced by the constraint $h_{2}$, defined below.

The responses evaluated during the optimization, such as the gripper position and the motor position, are obtained with the simulation of the flexible multibody with its embedded PID controller (Eqs. (3)-(6)).

Two set of constraints are imposed to avoid infeasible gains $(\leqslant) 0$ and large actuation forces $(\geqslant 500 \mathrm{~N})$ :

$h_{1}(\mathbf{s}): \mathbf{s}_{l} \leqslant \mathbf{s} \leqslant \mathbf{s}_{u}$

$h_{2}(\mathbf{s}): f_{2} \leqslant g_{u}$

where $\mathbf{s}_{l}$ and $\mathbf{s}_{u}$ assure that the control gains are always positive and that the structural parameter varies according to the available commercial options (from 0.02 to $0.04 \mathrm{~m}$ ); and $g_{u}$ is 1 guaranteing a maximum required force below $500 \mathrm{~N}$.

A set of constraints is imposed to guarantee that the system is uniformly asymptotically stable for a parameter variations between 0.33 and $0.53 \mathrm{~m}$ and bounded rate by $10.0 \mathrm{~m} / \mathrm{s}$ :

$h_{3}(\mathbf{s}): \begin{cases}\mathbf{P}(l(i))>0 & i=1, \ldots, 40 \\ \mathbf{A}(\mathbf{l}(i))^{T} \mathbf{P}(i+j) \mathbf{A}(\mathbf{l}(i))-\mathbf{P}(i)<0, & j=-1,0,1 \\ \mathbf{A}(\mathbf{l}(i+1))^{T} \mathbf{P}(i+j) \mathbf{A}(\mathbf{l}(i+1))-\mathbf{P}(i)<0, & \end{cases}$ 

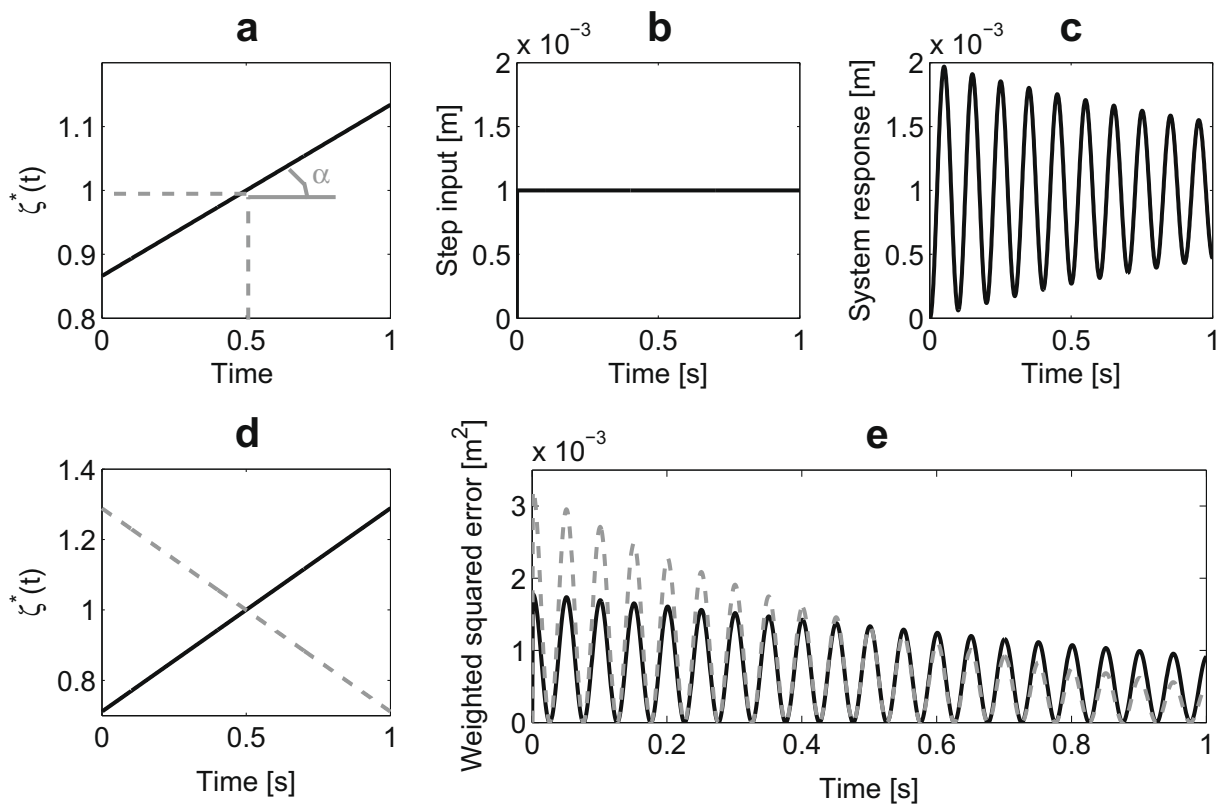

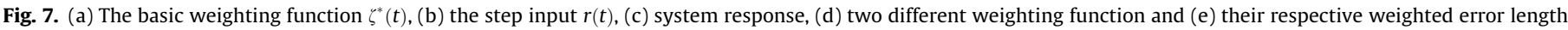
variation $l(t)$.

This constraint is evaluated in discrete-time; where $i$ represents the time step, $l$ is the beam length, $\mathbf{P}(l(i))$ are positive definite matrices and $\mathbf{A}(l(i))$ represented the discretized closed-loop state-space system (the reduced plant embedded with the controller).

For multi-objective optimization problems with three and four variables, described below, the adopted initial population and the population size are equal to 30 individuals (solutions); whereas for problems with seven variables, the initial population and the population size are equal to 40 individuals. Around 25 individuals are expected to be found on the Pareto-optimal solutions in both cases. The inverse crossover probability is chosen to be 0.85 , which guarantees the inclusion of new individuals in the optimization process. Finally, the maximum number of iterations is 30 , which is large enough to observe the algorithm convergence, which means that all individuals in the present population are dominated by the non-dominated individuals from the previous population.

Two control strategies and optimization problems are analyzed as follows:

Case 1 The gains of an LTI PID are optimized simultaneously with the diameter of the flexible beam. These results are compared with the nominal case $(d=24 \mathrm{~mm})$, where only the controller is optimized.

Case 2 The gains of an LPV gain-scheduling PID are optimized simultaneously with the diameter of the flexible beam. Comparisons between the integrated design considering the LTI PID and the LPV gain-scheduling PID controllers are reported.

\subsection{Case 1: integrated design considering an LTI PID controller}

Considering the reference input in Fig. 6, the gains of an LTI PID controller are optimized. Firstly, the nominal case $(d=24 \mathrm{~mm})$ is considered resulting in a 3 -variable optimization problem

$\mathbf{S}=\left\{K_{P}, K_{I}, K_{D}\right\}$

Secondly, the gains are optimized simultaneously with the beam diameter resulting in a 4 -variable optimization problem, i.e.

$$
\mathbf{s}=\left\{K_{P}, K_{I}, K_{D}, d\right\}
$$

The multi-objective optimization problem is stated as

$$
\begin{aligned}
& \min _{\mathbf{s}}\left[\begin{array}{l}
f_{1}(\mathbf{s}) \\
f_{2}(\mathbf{s})
\end{array}\right] \\
& \text { subject to }\left\{\begin{array}{l}
h_{1}(\mathbf{s}) \\
h_{2}(\mathbf{s}) \\
h_{3}(\mathbf{s})
\end{array}\right.
\end{aligned}
$$

Fig. 8 shows the Pareto-optimal solutions and Fig. 9 shows the values for the design variables associated with each individual on the Pareto-optimal solutions for both nominal case and integrated design. It can be observed from Fig. 8, that the inclusion of the structural variable improves the overall design. For instance, considering the same $f_{1}$ value (see the squares in Figs. 8 and 9) and increasing slightly the beam diameter (12.5\%), the value of $f_{2}$ is considerably reduced (from 0.58 to 0.35 ). This reduction actually means that the maximum required force by the controller is lower than the nominal case, i.e. the same level of performance can be achieved with a smaller motor. On the other hand, considering the same $f_{2}$ value (see the circles in Figs. 8 and 9) and increasing the beam diameter, the value of $f_{1}$ is considerably reduced. In general, the

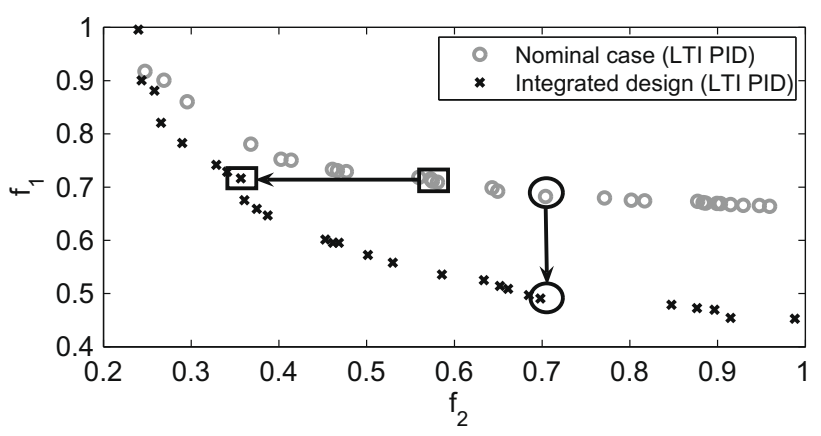

Fig. 8. Pareto-optimal solutions considering the LTI PID: (o) nominal case and (x) integrated design. 

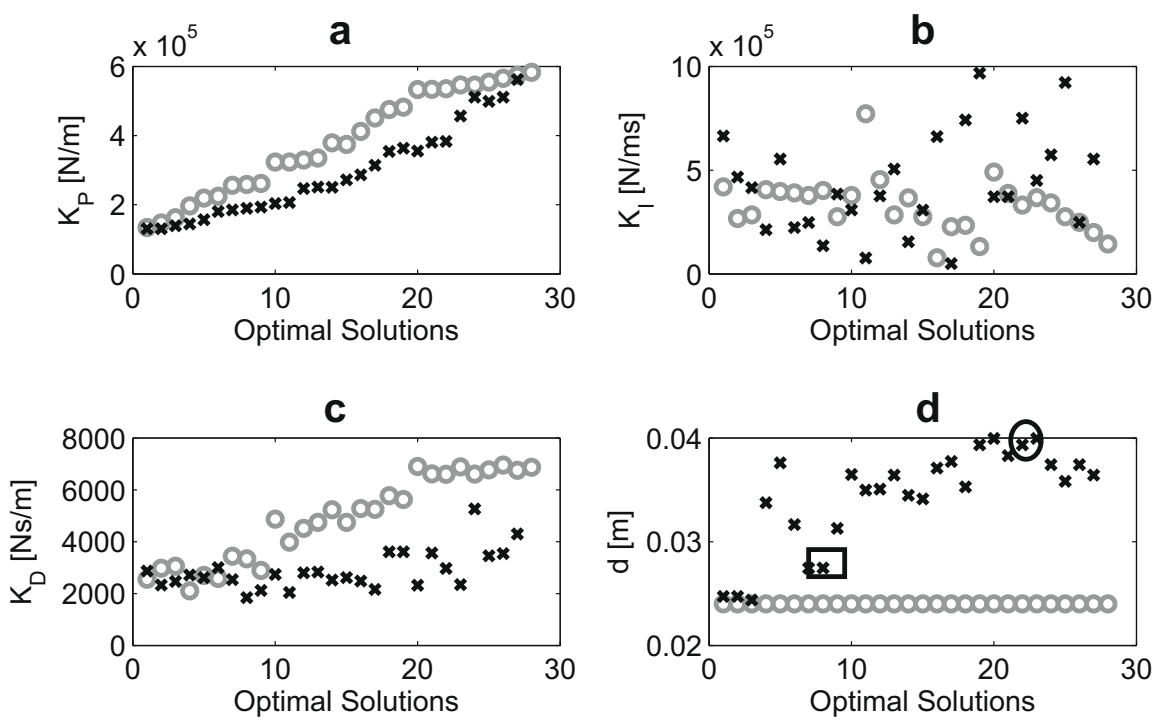

Fig. 9. Optimal solutions considering the LTI PID: (o) nominal case and ( $\mathrm{x}$ ) integrated design.

optimal integrated solutions have resulted in thicker diameters (see Fig. 9d). However, since the maximum force required by the system is related to both beam diameter and PID gains, thicker diameters are not always yielding higher values of the maximum force (see the squares in Figs. 8 and 9). Moreover, the design constraint, $h_{2}$, is respected in the whole objective space, which means that the present motor can be kept and the maximum required force is below $500 \mathrm{~N}$.

\subsection{Case 2: integrated design considering an LPV gain-scheduling PID controller}

Considering the reference input in Fig. 6, the gains of an LPV gain-scheduling PID and the beam diameter are optimized simultaneously. This optimization problem leads to seven design variables

$\mathbf{s}=\left\{K_{P 0}, K_{P 1}, K_{I 0}, K_{I 1}, K_{D 0}, K_{D 1}, d\right\}$

As performed previously (Eq. (24)), both objective functions $f_{1}$ and $f_{2}$ are considered. Fig. 10 shows the Pareto-optimal solutions for the aforementioned LTI PID controller (case 1) and for the LPV gain-scheduling PID controller (case 2). It can be observed that the LPV gain-scheduling PID can perform slightly better than the LTI PID, since the solutions of the latter are located below the solutions of the former. The variations of the optimal beam diameter along the Pareto front are illustrated in Fig. 11. There is no significant difference between the solutions set.

It can be observed from Figs. 10 and 11 that the LPV gain-scheduling performance and solution sets are rather similar to the per-

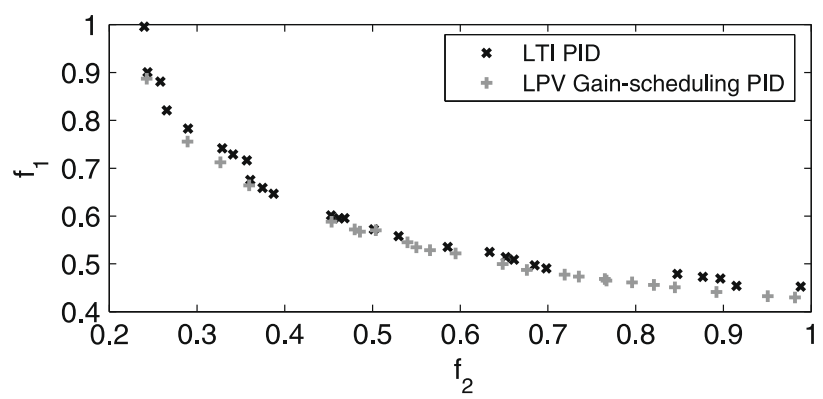

Fig. 10. Pareto-optimal solutions of the integrated design considering the LTI PID and the LPV gain-scheduling PID controllers.

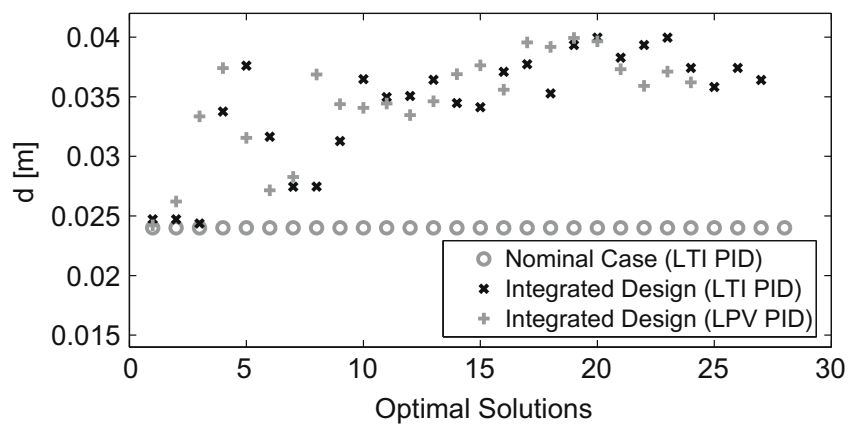

Fig. 11. Optimal beam diameters: nominal case and integrated design considering the LTI PID and the LPV gain-scheduling PID controllers.

formance and solution set presented by the LTI PID. The main reason for this is because the optimal beam diameters are rather larger than the nominal (see Fig. 11); therefore, less sensitive to vibrations. In other words, the amplitude of these vibrations are small over the whole configuration space. Therefore, the performance of the LPV gain-scheduling PID controller, considering a linear interpolation, does not present much improvement when compared with the LTI PID controller.

The design procedure proposed here allows this kind of analysis, as it enables the evaluation of continuous machine operation in closed-loop, with different control and structure configurations. As a result, for the chosen set and range of parameters, it can be concluded that the use of a thicker beam diameter, if affordable, can lead to a simpler controller configuration without loss in accuracy.

\section{Conclusions}

This paper addresses the integrated design of structural and control parameters of serial machines with flexible components. A multi-objective optimization framework has been developed based on a general simulation tool for flexible multibody systems embedded with nonlinear controllers.

The methodology is exploited for the optimization of a pickand-place assembly robot with a gripper carried by a variablelength flexible beam. The model involves a sliding joint that 
connects the flexible beam to the rigid frame. The beam diameter and the gains of LTI and LPV gain-scheduling PID controllers are optimized according to a direct design strategy.

These results reveal the benefits of the mechatronic design approach since the active system design tradeoffs are identified. The qualitative statement that the optimal integrated solutions result in thicker diameters seems to be predictable. Actually, any thicker diameter would imply in vibration reduction. However, the quantitative results achieved in this framework are not that simple to foresee. Using the proposed methodology, one can decide which beam diameter should be selected and predict the closed-loop response of such a complex mechatronic system in time-domain. In this way, not only qualitative behavior, but also quantitative metrics, such as overshoot and settling, can be evaluated over the configuration space during the design phase.

For future work, tighter performance requirements could be achieved using more advanced control strategies, such as robust and optimal controllers, with the nested design strategy.
(41 configurations from $l=0.33-0.53 \mathrm{~m}$ ). This appendix reports the reduced model for $l=0.53 \mathrm{~m}$. The reduced mass and stiffness matrices are built according to a selected set of modes: 1 rigidbody mode and 3 kept flexible modes, yielding 4 by 4 matrices. A state-space model can be derived from the reduced mass and stiffness matrices, the modal matrices and the chosen modal damping factor. Eventually, the flexible multibody has been reduced from hundreds degrees-of-freedom to a state-space model with 8 states, 1 input (the motor force) and 2 outputs (the motor position and the gripper position). Details on the model-reduction technique and its application on the case study can be found in [5].

The minimal realization state-space model of this reduced model is given by the following equations:

$$
\begin{aligned}
& \dot{\mathbf{x}}_{p}=\mathbf{A}_{p}(0.53) \mathbf{x}_{p}+\mathbf{B}_{p}(0.53) \mathbf{u}_{p} \\
& \mathbf{y}_{p}=\mathbf{C}_{p}(0.53) \mathbf{x}_{p}+\mathbf{D}_{p}(0.53) \mathbf{u}_{p}
\end{aligned}
$$

where

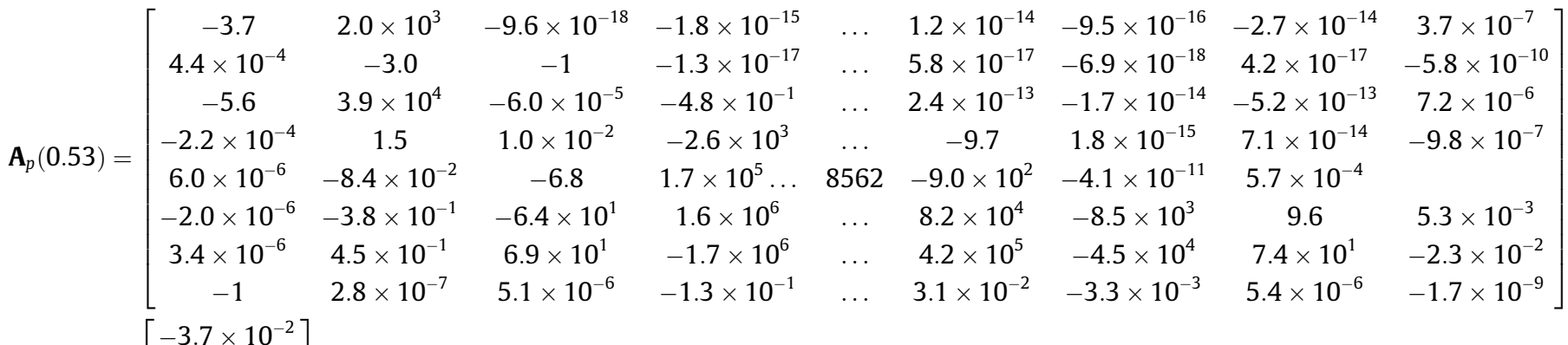

$$
\begin{aligned}
& \mathbf{B}_{p}(0.53)=\left[\begin{array}{c}
-3.7 \times 10^{-2} \\
4.4 \times 10^{-6} \\
5.6 \times 10^{-2} \\
-1.5 \times 10^{-5} \\
8.6 \times 10^{-3} \\
8.1 \times 10^{-2} \\
-8.3 \times 10^{-2} \\
-6.1 \times 10^{-9}
\end{array}\right] \\
& \mathbf{C}_{p}(0.53)=\left[\begin{array}{ccccccccc}
-2.5 \times 10^{-13} & 2.4 \times 10^{-7} & 3.9 \times 10^{-5} & -9.9 \times 10^{-1} & \ldots & -1.5 \times 10^{-1} & 1.6 \times 10^{-2} & -3.2 \times 10^{-5} & 1.0 \\
-2.5 \times 10^{-13} & -7.7 \times 10^{-1} & -1.0 \times 10^{-4} & 9.9 \times 10^{-1} & \ldots & 1.5 \times 10^{-1} & -1.6 \times 10^{-2} & 3.2 \times 10^{-5} & 1.0
\end{array}\right] \\
& \mathbf{D}_{p}(0.53)=\left[\begin{array}{l}
0 \\
0
\end{array}\right]
\end{aligned}
$$

\section{Acknowledgements}

The research of Maíra M. da Silva was supported by CAPES, Brazilian Foundation Coordination for the Improvement of Higher Education Personnel, and by the K.U.Leuven Research Council. The research presented in this paper was performed as part of the Marie Courie RTN project: A Computer Aided Engineering Approach for Smart Structures Design (MC-RTN-2006-035559) and the EU project: NEXT (IP 011815). The scientific responsibility is assumed by its authors.

\section{Appendix A}

The model-order reduction technique described in [27] has been applied to the full flexible multibody model, described in Section 4.1 , for each configuration considered in the stability analysis

\section{References}

[1] Van Amerongen J. Mechatronic design. Mechatronics 2003;13:1045-66.

[2] Lu J, Skelton RE. Integrating structure and control design to achieve mixed $\mathrm{H}_{2} / \mathrm{H}_{\infty}$ performance. Int J Control 2000;73(16):1449-62.

[3] Wujun F, Changming Z. Active damping guide roller design based on integrated structure/controller optimization with a structured controller. In: Proceedings of IDETC/CIE 2005, ASME 2005 international design engineering technical conferences and computers and information in engineering conference, Long Beach, USA; September 24-28 2005

[4] Rieber JM, Taylor DG. Integrated control system and mechanical design of a compliant two-axes mechanism. Mechatronics 2004;14(9):1069-87.

[5] da Silva MM, Brüls O, Swevers J, Desmet W, Van Brussel H. Computer-aided integrated design for machines with varying dynamics. Mech Mach Theory 2009;44(9):1733-45.

[6] Ravichandran T, Wang D, Heppler G. Simultaneous plant-controller design optimization of a two link planar manipulator. Mechatronics 2006;16(3-4): 233-42.

[7] Affi Z, EL-Kribi B, Romdhane L. Advanced mechatronic design using a multiobjective genetic algorithm optimization of a motor-driven four-bar system. Mechatronics 2007;17:489-500. 
[8] De Oliveira LPR, Da Silva MM, Sas P, Van Brussel H, Desmet W. Concurrent mechatronic design approach for active control of cavity noise. J Sound Vibr 2008;314(3-5):507-25.

[9] Jianwei L. Study on the integrated structural design and noise attenuation of flexible linkage mechanism. Mechatronics 2008;18(3):153-8.

[10] Apkarian P, Gahinet P, Becker G. Self-scheduled $H_{\infty}$ control of linear parameter-varying systems: a design example. Automatica 1995;31: 1251-61.

[11] Van Brussel H, Sas P, Németh I, De Fonseca P, Van den Braembussche P. Towards a mechatronic compiler. IEEE/ASME Trans Mech 2001;6(1): 90-105.

[12] Wang X, Mills JK. Dynamic modeling of a flexible-link planar parallel platform using substructuring approach. Mech Mach Theory 2006;41:671-87.

[13] da Silva MM, Desmet W, Van Brussel H. Design of mechatronic systems with configuration-dependent dynamics: simulation and optimization. IEEE/ASME Trans Mech 2008;13(6):638-46.

[14] Zaeh M, Siedl D. A new method for simulation of machining performance by integrating finite element and multi-body simulation for machine tools. Ann CIRP 2007:6(1):383-6.

[15] Craig RR. A review of time domain and frequency domain component mode synthesis methods. In: Proceedings of the joint mechanics conference, Albuquerque, USA; June 24-26 1985.

[16] Cardona A, Klapka I, Géradin M. Design of a new finite element programming environment. Eng Comput 1994;11:365-81.

[17] Géradin M, Cardona A. Flexible multibody dynamics: a finite element approach. Chichester: John Wiley \& Sons; 2001.

[18] Brüls O, Golinval JC. The generalized- $\alpha$ method in mechatronic applications. J Appl Math Mech 2006;86(10):748-58.
[19] Samin JC, Brüls O, Collard JF, Sass L, Fisette P. Multiphysics modeling and optimization of mechatronic multibody systems. Multibody Syst Dyn 2007;18(3):345-73.

[20] Paijmans B, Symens W, Van Brussel H, Swevers J. A gain-scheduling control technique for mechatronic systems with position-dependent dynamics. In: Proceedings of American control conference, Minneapolis, USA; June 14-16 2006.

[21] Amato F, Mattei M, Pironti A. Gain scheduled control for discrete-time systems depending on bounded rate parameters. Int J Robust Nonlin Control 2005;15:473-94

[22] Paijmans B. Interpolating gain-scheduling control for mechatronic systems with parameter-dependent dynamics. PhD Thesis, Katholieke Universiteit Leuven; 2007.

[23] Srinivas N, Deb K. Multi-objective function optimization using non-dominated sorting genetic algorithms. J Evolut Comput 1994;2(3):221-48.

[24] Deb K, Agrawal S, Pratap A, Meyarivan T. A fast elitist non-dominated sorting genetic algorithm for multi-objective optimization: NSGA-II. IEEE Trans Evolut Comput 2002;6(2):182-97.

[25] da Silva MM. Computer-aided integrated design of mechatronic systems. PhD Thesis, Katholieke Universiteit Leuven; 2009.

[26] De Caigny J, Demeulenaere B, De Schutter J, Swevers J. Polynomial spline input design for LPV motion systems. In: Proceedings of 10th IEEE international workshop on advanced motion control, Trento; March 26-28 2008.

[27] Brüls O, Duysinx P, Golinval JC. The global modal parameterization for nonlinear model-order reduction in flexible multibody dynamics. Int J Numer Meth Engng 2007;69:948-77.

[28] Gahinet P, Nemirowski A, Laub AJ, Chilali M. LMI control toolbox. Natick, MA: The mathworks, 1995. 